\title{
Childhood lichen planus pigmentosus
}

\section{Monia Youssef', Lahouel Ines ${ }^{1}$, Marmouch Héla², Rim Hadhri ${ }^{3}$, Hayet Akkari', Mariem Mohamed', Yosra Soua', Hichem Belhadjali' ${ }^{1}$, Jameleddine Zili'}

\author{
${ }^{1}$ Department of Dermatology, Fattoum Bourguiba Hospital, University of Medicine, Monastir, Tunisia, ${ }^{2}$ Department of \\ Endocrinology, Fattouma Bourguiba Hospital, University of Medicine, Monastir, Tunisia, ${ }^{3}$ Department of Anatomopathology, \\ Fattoum Bourguiba Hospital, University of Medicine, Monastir, Tunisia
}

Corresponding author: Dr. Lahouel Ines, MD, E-mail: lahouel.iness@gmail.com

Sir,

Lichen planus pigmentosus (LPP) is a rare variant of lichen planus that presents as asymptomatic to mildly pruritic, hyperpigmented macules and/or patches. This dermatosis has been rarely reported in children. We report a first series of childhood LPP illustrating 5 cases from Tunisia.

Of 5 patients (age range 6-16 years), 3 were male (mean age, 9.3 years) and 2 were females (mean age, 13 years). The duration of disease at presentation ranged from 1 month to 1 year. In 4 patients, lesions were present at more than one anatomical site and the patients had bilateral involvement. The trunck was the commonest site affected (4 cases). In our all cases, the lesions were a few $\mathrm{mm}$ to a few $\mathrm{cm}$ in diameter (Fig. 1). The various colour shades noted were dark brown in four cases and slate grey in one patient. The pattern of pigmentation was diffuse in all patients. In 4 patients, the lesions were asymptomatic. One patient had mild itching. Oral mucosa was involved in only one patient, who had lesion localized in the lips. This patient had brown pigmentation present over the buccal mucosa (gingivae) of both sides. Palms, soles and nails were spared in all cases. None of the patients presented coincidental lichen planus.

No elevated active red border as seen in erythema dyschromicum perstans was noticed in any patient. The history of previously inflammatory processes in affected areas was not present. All cases had no concomitant diseases. Related external factors such as drug intake prior to the onset or use of cosmetics were absent. Our second patient reported that lesions appeared after the exposure to a severe stress. None

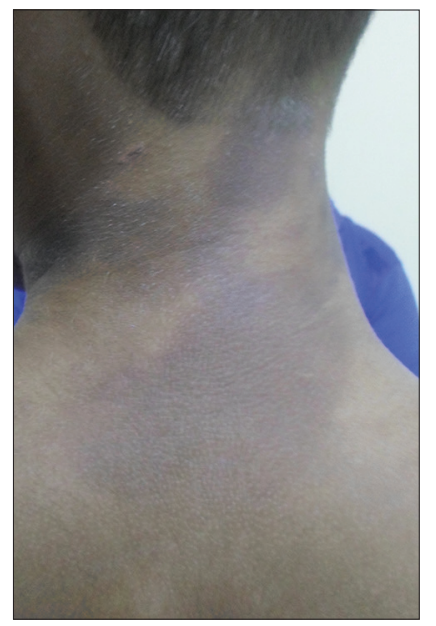

Figure 1: Pigmented brownish, well-defined macules located on the neck.

of our patients had a family history of a similar skin disorder.

Of the 4 patients, who were treated with topical betamethasone, two showed a slight lightening of the pigmentation after an average of 16 weeks.

Skin biopsies were performed from all the patients. Three patients had band-like lymphocytic infiltrate and two patients had superficial perivascular pattern. Melanin incontinence was a constant finding in all cases. Hyperkeratosis was marked in three cases and keratinocyte necrosis in one case.

The clinical, histological and therapeutic features of our patients are summarized in Table 1.

We reported 5 Tunisian cases of childhood LPP, a rare entity in children. LPP is a rare variant of lichen planus, 
Table 1: Clinical, histological, and therapeutic features of patients

\begin{tabular}{|c|c|c|c|c|c|c|}
\hline No. of cases & Sex/age & Duration & Site & Histological examination & Treatment & Course \\
\hline Case1 & $\mathrm{M} / 6$ & 4 months & Neck Trunck & $\begin{array}{l}\text { Perivascular lymphohistiocytic } \\
\text { infiltrate, pigmentary incontinence, }\end{array}$ & Topical betamethasone & No improvement \\
\hline Case2 & $\mathrm{F} / 10$ & 1 year & $\begin{array}{l}\text { Upper and lower } \\
\text { limbs, trunck }\end{array}$ & $\begin{array}{l}\text { Hyperkeratosis, band-like lymphocytic } \\
\text { infiltrate, pigmentary incontinence }\end{array}$ & Topical betamethasone & Slight improvement \\
\hline Case 3 & $M / 13$ & 1 month & $\begin{array}{l}\text { Trunck, Upper } \\
\text { limbs }\end{array}$ & $\begin{array}{l}\text { Hyperkeratosis, pigmentary } \\
\text { incontinence, band-like lymphocytic } \\
\text { infiltrate, keratinocyte necrosis }\end{array}$ & Topical betamethasone & No improvement \\
\hline Case4 & $\mathrm{F} / 16$ & 3 months & $\begin{array}{l}\text { Lips,Oral } \\
\text { mucosa }\end{array}$ & $\begin{array}{l}\text { Hyperkeratosis, pigmentary } \\
\text { incontinence, melanophages, } \\
\text { band-like lymphocytic infiltrate }\end{array}$ & Photoprotection & No improvement \\
\hline Case 5 & $M / 9$ & 6 months & $\begin{array}{l}\text { Neck, trunck, } \\
\text { lowerlimbs }\end{array}$ & $\begin{array}{l}\text { Perivascular lymphohistiocytic } \\
\text { infiltrate, pigmentary incontinence }\end{array}$ & Topical betamethasone & Slight improvement \\
\hline
\end{tabular}

first described by Bhutani et al [1]. It generally starts in the third or fourth decade of life and there is a slight female predilection [2]. The prevalence of LPP among children with lichen planus is low ranging from 2.8 to $4 \%[3,4]$.

LPP is characterized by the presence of the hyperpigmented, dark-brown macules in the sun exposed or flexural areas of the body. Face and neck are the most frequent initial sites of involvement. Palms, soles and nails are not affected. Although lesions are generally asymptomatic, mild pruritus and burning sensations are present in about one-third of patients [2]. In our pediatric series, the trunck was the most common site of lesions, itching was present in only one patient.

Our fourth patient presented oral lesions. In fact, while Bhutani et al [1] stressed that LPP was never localized in the oral mucosa. Laskaris et al [5] described a case of LPP of the oral mucosa. To our Knowledge, this rare clinical variety had never been reported before in children.

Histopathological examination typically shows vacuolar alteration of the basal layer, variable dense of lymphocyte infiltration, pigmentary incontinence and melanophages in the superficial dermis. In our cases we have found similar histopathological results, as well.

The differential diagnosis of childhood LPP includes idiopathic eruptive macular pigmentation, ashy dermatosis and post-inflammatory pigmentation.

The etiopathogenesis of the condition remains unknown. Kanwar et al suggested that mustard oil, amla oil, henna and hair dye could be the precipitating factors in predisposed individuals [2]. These factors could not be identified in any of our patients. Nevertheless, the exposure to a severe stress might be a precipitating factor in one of our patients.

The disease is insidious in onset and has a chronic course [2]. The treatment is based on corticosteroids and topical tacrolimus. The skin lesions are often resistant to treatment, as evidenced by our observations.

To conclude, LPP in childhood is uncommon. As this was a first series of childhood LPP. It confirms the literature data when the scarcity of the entity and the disappointing response to treatment. Mucosal involvement might be not infrequent. However, additional studies are needed to better characterize this entity in children.

\section{REFERENCES}

1. Bhutani LK, Bedi TR, Pandhi RK, Nayak NC. Lichen planus pigmentosus. Dermatologica. 1974;149:43-50.

2. Kanwar AJ, Dogra S, Handa S, Parsad D, Radotra BD. A study of 124 Indian patients with lichen planus pigmentosus. Clin Exp Dermatol. 2003;28:481-5.

3. Pandhi D1, Singal A, Bhattacharya SN. Lichen planus in childhood: a series of 316 patients. Pediatr Dermatol. 2014;31:59-67.

4. Nanda A1, Al-Ajmi HS, Al-Sabah H, Al-Hasawi F, Alsaleh QA. Childhood lichen planus: a report of 23 cases. Pediatr Dermatol. 2001;18:1-4.

5. Laskaris GC, Papavasiliou SS, Bovopoulou OD, Nicolis GD. Lichen planus pigmentosus of the oral mucosa: a rare clinical variety. Dermatologica. 1981;162:61-3.

Copyright by Monia Youssef, et al. This is an open access article distributed under the terms of the Creative Commons Attribution License, which permits unrestricted use, distribution, and reproduction in any medium, provided the original author and source are credited.

Source of Support: Nil, Conflict of Interest: None declared. 